\title{
The interplay between affective and cognitive factors in shaping early proficiency in mathematics ${ }^{\text {in }}$
}

\author{
Elisa Cargnelutti ${ }^{\mathrm{a}}$, Carlo Tomasetto ${ }^{\mathrm{b}}$, Maria Chiara Passolunghi ${ }^{\mathrm{c}, *}$ \\ a Department of Life Sciences, Unit “Gaetano Kanizsa”, University of Trieste, via Weiss 21, 34128 Trieste, TS, Italy \\ b Department of Psychology, University of Bologna, Piazza Aldo Moro, 90, 47521 Cesena, FC, Italy \\ " Department of Life Sciences, Unit "Gaetano Kanizsa”, University of Trieste, via Weiss 21, 34128 Trieste, TS, Italy
}

\section{A R T I C L E I N F O}

\section{Keywords:}

General anxiety

Math anxiety

Working memory (WM)

Approximate number system (ANS)

Cognitive precursors

Numerical cognition

\begin{abstract}
A B S T R A C T
Performing math tasks is a complex process that requires the recruitment of many cognitive and affective factors. Research on the interplay between cognitive and affective factors associated with math ability is surprisingly scarce in primary school children. In the present study, we examined the contribution of both general and mathspecific anxiety to math performance in a large sample of second-grade schoolchildren, and also their relation with different measures of both domain-general (i.e., spatial and verbal working memory, intelligence) and domain-specific cognitive correlates of math ability (i.e., different skills tapping the approximate number system, ANS). Results revealed a negative relation between general anxiety (but not math anxiety) and math performance, beyond the contribution of the cognitive abilities. Importantly, specific components of both verbal working memory (i.e., digit span) and ANS (i.e., approximate addition) mediated the relation between general anxiety and math performance. The educational implications of these findings are discussed.
\end{abstract}

\section{Introduction}

Learning a discipline such as mathematics is a complex process to which many different factors concur. Also for this reason, in this domain there is a high prevalence of learning difficulties (see [8]), which lead to negative effects that reverberate not only on the academic success or future career expectations (see [75] for a review), but also on the individual's well-being (e.g., [44]). It is therefore not surprising that intensive research (e.g., $[20,23,33,118]$ ) is devoted to the detection of the cognitive factors that prompt math proficiency, or, when deficient, underlie math learning disabilities (MLD).

Similarly, a growing amount of studies has also stressed the importance of the affective factors as determinants of math achievement, with a prevailing focus on the role of anxiety (e.g., [17,50,82]). However, only a few studies to date have jointly investigated the role of both cognitive and affective factors associated with math ability, and even fewer have focused on young children (e.g., [43,70,96,97,111]). To address this gap in the literature, in the present study we examined the interplay between both domain-general and domain-specific cognitive and affective factors at the basis of math ability in a young sample of second-grade schoolchildren.

\section{Cognitive correlates of math proficiency}

\subsection{Domain-general components: working memory}

The domain-general cognitive correlates of math include the cognitive abilities, such as memory and intelligence, that support the individuals' proficiency not only in math, but also in a variety of other domains including language (e.g., $[34,86]$ ). It is generally assumed, for instance, that intelligence is a relevant domain-general math correlate (e.g., $[38,60,84,87])$. However, it should be noted that the intellectual profile of children with math disabilities is not compromised: Individuals affected by MLD have by definition intelligence levels within the normal range (see DSM-V, [1]).

Beyond general intelligence, memory is probably the most studied domain-general correlate of math. Consistent with the widely accepted Baddeley's model ([5,6]; see also [37]), memory systems may be subdivided in a more passive component, essentially devoted to transient information retention and precisely expressed in terms of short-term memory (STM), and in a more active component, defined as working memory (WM) in stricto sensu, and additionally involved in information elaboration. According to this model, STM is represented by the two stores of the phonological loop and of the visuo-spatial sketchpad,

\footnotetext{
Affiliation where the research was conducted: Department of Life Sciences, University of Trieste, TS, Italy.

* Correspondence to: Maria Chiara Passolunghi, Department of Life Sciences, University of Trieste, via Weiss 21, 34128 Trieste, TS, Italy.

E-mail addresses: eli.cargnel@gmail.com (E. Cargnelutti), carlo.tomasetto@unibo.it (C. Tomasetto), passolu@units.it (M.C. Passolunghi).
} 
whereas WM coincides with the so called central executive. Whereas WM is almost unanimously viewed as a key contributor of math proficiency (e.g., $[15,37,57,84]$; Peng et al., 2016), the relative importance of specific verbal and visuo-spatial memory components is still debated (see [21]). Although both components appear related to math proficiency, it remains unclear whether verbal and visuo-spatial components may differently contribute to the diverse math domains (e.g., calculation vs. geometry; for recent meta-analyses, see Friso-van den Bos et al., 2013; [92]), and whether their relation with math proficiency may vary according to the children's stage of development (see [52,76,77]). Importantly, not only the WM domain, but also the content of the tasks used to assess verbal WM may influence the relation with math skills. Namely, it has been suggested that a stronger association with math proficiency may appear when tasks involving numbers are used to assess verbal WM (typically, backward digit recall; $[4,80,85,92]$ ). Several works attempted to control for this potential confound by using both digit and word (or letter) recall tasks to assess verbal WM. However, results are not conclusive: Whereas some findings with children with math difficulties demonstrated specific impairments in WM tasks involving numerals (e.g., [39,51,89]), others did not (e.g., [107]; see Peng et al. 2016 for a meta-analysis).

\subsection{Domain-specific components: approximate number system (ANS)}

Beside the domain-general abilities, research has intensively focused on the role of basic skills tightly pertaining to the numerical domain, and hence defined as domain-specific correlates of numeracy. These include, among all, the innate ability of estimating and comparing quantities and magnitudes in an approximate manner and are commonly referred to as the approximate number system (ANS; e.g., $[65,74])$. Here, we precisely consider ANS by accounting for the very basic ability of judging quantities expressed in the non-symbolic (e.g., dots) rather than in symbolic (i.e., Arabic numerals) format.

The acuity of non-symbolic ANS has been proven to be an important predictor of math learning in young children, prior to accessing formal math teaching $[55,65,66,74,84]$. On the other hand, many authors argued that these abilities have a minor role when symbolic and exact numerical skills are taken into account (e.g., [11,53,102,109]), despite a few evidences of a still significant ANS role in older children and adults (e.g., $[31,67,91]$ ).

A relevant issue regarding ANS deals with the tasks developed to assess its acuity. Probably the most widely used task tapping into ANS is represented by approximate comparison, which requires children to compare two arrays of elements (e.g., dots), in order to rapidly judge which is the most numerous (see [46]). In order to control for potential confounds due to the covariations between numerosity and irrelevant perceptual features of the stimuli (e.g., size, density, etc.), these tasks have to include perceptual constraints that inevitably pose additional cognitive demands when performed (e.g., the most numerous array is made up by dots of smaller size). Hence, inhibition skills are required to focus on numerosity without being biased by the perceptual constraints (for a detailed explanation on the set up of the ANS tasks, see [25]).

For this reason, some authors (e.g., [35,41,105]) cautioned that ANS acuity could come out as a significant predictor of formal math ability because it is, at least in part, a measure of inhibition. Nevertheless, interindividual variability in ANS acuity is documented also in adults, for whom the ANS tasks are expected to be less demanding in terms of inhibitory control (e.g., [45,67]).

To address these concerns, several investigations have combined two or more alternative tasks to assess ANS, beside the dot comparison task (e.g., approximate addition tasks; [12,56]). Findings from these studies were also contradictory, with only low-to-moderate correlations among ANS tasks, and inconsistent associations between different ANS tasks and formal numeracy (see $[24,40]$, for reviews). Inconsistencies across studies may in part depend on the participants' age, as some ANS aspects more than others may be indicative of math performance across grades or ages (e.g., [95,118]). Pinheiro-Chagas et al. [95] also suggested that a sort of hierarchy between diverse ANS components may exist, as approximate addition and estimation were found to mediate the relation between approximate dot comparison and exact calculation in middle childhood.

\subsection{Affective factors: general and math-specific anxiety}

Anxiety is commonly defined as an aversive emotional and motivational state in response to environmental threats, and general (or trait) anxiety refers to the degree with which individuals experience current situations as threatening [28]. As regards mathematics, a specific form of anxiety has been identified and referred to as math anxiety (for a comprehensive review, see [27]). Math anxiety consists in a feeling of apprehension and fear associated to the actual or anticipated manipulation of the numerical information (see Hembre, 1990). Math anxiety has been identified as dissociable from general anxiety from as early as 7 to 9 years of age [119], with specific and derailing effects on math proficiency also in children affected by MLD (e.g., [73,100,117]).

The relation between anxiety (both general and math-specific) and math proficiency in young children is however still debated. A dearth of literature reports general evidence of decreased performance in presence of high levels of anxiety (e.g., [50,82]). This relation is evident also in children, especially with reference to general anxiety and when multi-informant approaches are adopted and teachers' reports are used to assess children's anxiety levels (e.g., [58,64,68,101]).

With regard to math-specific anxiety, evidence is more contradictory, and the existence of any relations between math anxiety and math proficiency in young children is still matter of debate. Actually, some findings suggest that a significant relation between math anxiety and math performance may already exist in the very first primary school grades [106,111,115,117,96,97], whereas other studies do not support this early relation (e.g., [61]). Some authors also proposed that the relation may attain statistical and practical relevance only gradually, through the subsequent school grades (Cargnelutti et al., 2017; [108]). Cargnelutti et al. (2017), for example, demonstrated that the concurrent (negative) relation between general anxiety and math performance was significant in second grade, whereas the contribution of math-specific anxiety was not. However, math anxiety in second grade indirectly predicted math performance one year later, thus suggesting that the affective correlates of math should be carefully monitored from the earliest school grades.

\subsection{The relation between cognitive and affective factors}

The interference of high levels of anxiety on the execution of demanding cognitive tasks is well established, and different theoretical models have been proposed to account for the observed relation between anxiety and cognitive proficiency (e.g., [29]). For instance, a central tenet of the attentional control theory [29] is that anxiety decreases processing efficiency by deviating attention from the task at hand towards threat-related cues, which occupy the memory compartments (especially WM). This phenomenon was observed also specifically for math. According to the inhibition mechanism theory [54], in fact, math performance impairment has to be attributed to the onset of math specific anxiety, which similarly to general anxiety absorbs the WM resources in order to suppress the competing worrisome thoughts (see [16]). Not surprisingly, the negative effects of math anxiety on cognitive proficiency were shown to increase in parallel with the WM demands of the task [3], and a lower WM performance was observed in children with high math anxiety [83].

Although the relation between anxiety and WM is well established, far less explored is the link between anxiety and the other cognitive abilities at the basis of math, such as ANS. Moreover, prior studies almost exclusively investigated the relation of ANS with math anxiety-rather than general anxiety-, and primarily took into account the 
symbolic ANS component. For instance, a decreased performance in symbolic magnitude processing was observed in high-math anxious adults by Núñez-Peña and Suárez-Pellicioni [81] and by Maloney and colleagues [71,72], who additionally found that WM intervened as a mediator in the relation between symbolic skills and math anxiety. In contrast, no significant association emerged between math anxiety in the few studies that adopted non-symbolic tasks to assess magnitude processing in children $[43,47]$. Finally, in a study with young adolescents, none significant relation with math anxiety surfaced either with symbolic or non-symbolic estimation acuity [112]. Evidence with this regard is hence mixed. In addition, to the best of our knowledge, no one study thus far explored the relation between anxiety and non-symbolic ANS acuity in children younger than 8 .

\subsection{The current study}

The core purpose of the present research was to achieve a more thorough view on the interplay between the cognitive and the affective factors that contribute to math proficiency in young schoolchildren. Our first goal was therefore to determine the unique and independent contribution of each of these diverse factors to early math performance. Second, we aimed to inspect whether the cognitive abilities (and, in case, which of them) might intervene as mediators in the relation between anxiety and math performance. Hence, we sought to verify whether anxiety-both general and math-specific- might disrupt math proficiency not only by interfering with the domain-general factors, such as WM, but also by disrupting the acuity of the domain-specific ANS components. To the best of our knowledge, this was the first study to explore the interplay between both general and math anxiety not only with WM, but also with the non-symbolic ANS abilities in the definition of math proficiency. As the research conducted thus far to address these issues in the earliest school grades is particularly sparse, we chose to target children attending Grade 2.

In detail, concerning the cognitive factors, we assessed diverse WM and ANS components. In this way, we attempted to understand whether possible discrepancies across previous studies might be due to the evaluation of different components within these constructs. For this reason, in relation to memory, we investigated both its verbal and visuo-spatial components. In reference to the former, we separately assessed the recall of both numbers (digits) and words. We opted to focus exclusively on WM, basically for the reason that-contrary to STM-it involves high-control processes, which are both strongly relevant to math learning and also selectively impaired by anxiety (e.g., $[3,30]$ ). Regarding ANS, we included three different tasks tapping into different facets of non-symbolic magnitudes processing (i.e., approximate comparison, approximate addition, and numerosity estimation). Finally, we assessed intelligence in order to ensure that our findings might hold even when controlling for the influence of individual differences in the general intellectual level.

We hypothesized that anxiety (especially general anxiety, at least in children of this age) might significantly contribute to math performance above and beyond the cognitive factors. We expected that it might be also indirectly related to math, through the negative effect on both the domain-general and the domain-specific cognitive abilities that support math performance.

\section{Method}

\subsection{Participants}

One hundred and sixty one Grade 2 children were recruited for the study. Children attended eight different classes across five primary schools in Northeastern Italy. From the initial sample, three children were excluded because of diagnosed neurological diseases, and two for atypical age for their grade. The resulting sample included 156 children (72 females; mean age 7 years 7 months). An a priori sample size calculation using the $\mathrm{G}^{*}$ Power software indicated that a sample size of 137 participants would be required to detect a medium size effect in bivariate correlation analyses with power $=.95$ and $\alpha=.05$ (twotailed). The achieved power with 156 participants was .97 .

\subsection{Procedure}

Formal consent was obtained from the school headmaster, math teachers and students' parents. The study took place in the second term of Grade 2. Anxiety and math skills were investigated by collectively administering the related tests to the whole class, whereas the cognitive precursors were individually assessed in a quiet room outside the classroom. Teachers' ratings of children's general anxiety were also collected, as adults' observation of children's anxiety in relevant contexts (e.g., at school) were proven to provide a more robust and reliable assessment than the self-reports from children themselves, at least until the age of 8-9 years (e.g., [79]; see [22], for a meta-analysis). To the contrary, math anxiety is a more specific and context-dependent construct, that appears to be reliably and validly assessed through ageappropriate self-report measures even in young children (e.g., $[36,97,96,115,117])$.

\section{Measures}

\subsection{Cognitive factors}

\subsubsection{Intelligence}

Intelligence was assessed by administering the Vocabulary and Block design subtests from the WISC-III scale ([113], Italian edition, 2006), respectively for the assessment of verbal and performance intelligence. These are the two subtests that most strongly correlate with the global IQ (i.e., IQ measured with the whole scale), and proven to be adequate for a global intelligence assessment [103].

\subsubsection{Vocabulary.}

This subtest entailed children to give exhaustive definitions of up to 30 selected words. Responses received $0-2$ points in relation to their exhaustiveness, giving a maximum of 60 points. Task reliability expressed by Cronbach's alpha was .74.

\subsubsection{Block design.}

This subtest required the reproduction of up to 12 bi-dimensional configurations by means of solid cubes in a predefined range of time. Scoring varied according to the correct reproduction time, with a maximum of 69 points. Task reliability expressed by Cronbach's alpha was .80 .

For both subtests, raw scores were converted into age-referenced scores ranging 1-19. By averaging these two age-referenced scores, we then computed a composite Intelligence score, which we used as a control variable.

\subsubsection{Working memory (WM): verbal component}

The following two tasks were administered to assess verbal WM.

Backward word recall (see [90]).

The task required children listening the experimenter while reading word lists of increasing length, and then reproducing them in the inverted order (from the last to the first word). The test included up to 14 trials, two for each of the seven difficulty levels (two- to eight- word spans). Each correct reproduction received 1 point, with a maximum score of 14 points. Task reliability expressed by Cronbach's alpha was .86 .

Backward digit recall (from WISC-III, [113], Italian edition, 2006). In this task, children were asked to listen to digit lists of increasing length to then reproduce them in the inverted order of presentation. The test consisted of 14 trials, two for each of the seven levels of increasing 
difficulty (two- to eight- digit spans). Each correct reproduction received 1 point, resulting in a maximum score of 14 points. Task reliability expressed by Cronbach's alpha was .85 .

\subsubsection{Working memory (WM): visuo-spatial component}

The Path dual task (adapted from [63]) required children to perform two concomitant tasks: Selective recall of the first step (square) of pathways of increasing length traced on a grid by the experimenter and the simultaneous performance of a secondary task (i.e., tap the hand on the desk when a target square was touched). One point was assigned only if the selective recall and the secondary task were both correctly performed, giving a maximum score of 8 points. Task reliability expressed by Cronbach's alpha was .81 .

\subsubsection{Approximate number system (ANS)}

Three tasks were administered to achieve a multifaceted assessment of ANS. In developing these tasks, we followed the recommendations reported in previous studies (e.g., [12,94]). In order to prevent children's responses from relying on irrelevant quantity parameters of the stimuli, we controlled for dot and array size, total area, and dot density.

Approximate magnitude comparison (adapted from [93]). Children were asked to detect the most numerous of the two array of dots simultaneously presented on the pc screen. For each trial, one of the arrays contained the reference number of dots (16), while the other contained the target number of dots $10-22,11$ and 21 excluded). The task included four trials for each of the 10 possible target numerosities, giving total number of 40 trials. To each correct response, 1 point was assign, resulting in a maximum score of 40 . Task reliability expressed by Cronbach's alpha was .62.

Approximate addition (adapted from [56]). Two arrays of blue dots were shown in succession on the left side of the screen and hidden one at a time behind a black occluder. Dots had to be approximately mentally summed and then compared to a third array of red dots appearing on the right. Children had to rapidly judge whether the final amount of blue dots was more or less numerous than the array of red dots. The total number of dots per trial was between eight and 25 , with proportions between the total number of blue vs. red dots being 4:5, 4:6, 4:7 and their reciprocals 5:4, 6:4, 7:4. The test consisted of 24 trials, four for each of the six possible proportions. Each correct response was assigned 1 point, with a maximum score of 24 points. Task reliability expressed by Cronbach's alpha was .65.

Estimation (see [9]). Children were required to provide the estimation of the number of dots appearing on the pc screen. Children were said that the maximum numerosity was 30 . Each estimation received 0-3 points according to the degree of approximation to the actual numerosity ( 3 points for a response within $10 \%$ of the actual numerosity, 2 points within $20 \%, 1$ point within $10 \%$, 0 points otherwise). The final maximum score was 87 points. Task reliability expressed by Cronbach's alpha was .82.

\subsection{Anxiety}

\subsubsection{General anxiety}

Depression and Anxiety in Youth Scale (DAYS, [79]; Italian ed., 1995), developed for youths from 6 years of age onward.

The scale includes a self- and a teacher-reported measure. Concerning the self-rating measure (hereafter Anxiety-Self), we partially amended the original scale by removing or re-formulating some statements (for instance, the statement "I want to kill myself" was removed). The administered scale included eight items with a four-point-response scale ranging from 0 (never) to 3 (always). The maximum score was 24 points. Scale reliability expressed by Cronbach's alpha was .60 .

The teachers' scale (hereafter Anxiety-Observed) was retained in the original version. The questionnaire includes seven statements with a dichotomous response modality (true or false). Responses indicating the presence of an anxiety symptom were scored 1 point. The maximum score was 7 points. Scale reliability expressed by Cronbach's alpha was .65 .

\subsubsection{Math anxiety}

The Scale for Early Math Anxiety (SEMA, translated and adapted from [116]) includes 20 items requiring children to image either to have to solve a given math problem or to be experiencing common situations occurring during math lessons; they had therefore to indicate the level of nervousness they would have felt if these conditions were truly happening. This version provided four instead of the original five response options: Not nervous at all, a little nervous, somewhat nervous, and very nervous. Each response was scored 0 (not nervous at all) to 3 (very nervous), giving a maximum score of 60 points. Scale reliability expressed by Cronbach's alpha was .87 .

\subsection{Math tasks}

Written computation (specifically developed by taking as reference the math textbooks, see Cargnelutti et al., 2017) comprises 16 arithmetic computations (additions and subtractions) to be solved in $10 \mathrm{~min}$. For each correct solution. 1 point was assigned, giving a maximum of 16 points. Task reliability expressed by Cronbach's alpha was .84 .

Word problems (from [42]) required children to solve six word problem questions involving additions and subtractions. Up to 1.5 points were assigned for each correct answer (1 point for the correct set out of the expression, plus . 5 for the correct execution of the computation). The maximum score was 9 points. Task reliability expressed by Cronbach's alpha was .69.

MAT-2, module Number (from [2]) includes 11 tasks assessing a wide spectrum of abilities (e.g., ranking numbers from the smallest to the greatest or decomposing numbers) to be performed in $20 \mathrm{~min}$. Each correctly solved exercise was scored 1 point, with a maximum score of 11 points. Task reliability expressed by Cronbach's alpha was .74.

The scores achieved in the three tasks were standardized and then averaged in order to compute a global Math composite score (Cronbach's alpha $=.78$ ).

\section{Results}

\subsection{Preliminary results}

The statistical analyses were performed by the PAW Statistics 21 statistical package. Descriptive statistics and bivariate correlations between the variables used in the subsequent analyses are reported in Table 1. All the included measures attained adequate reliability. Math score was significantly associated with all variables, except for Approximate magnitude comparison, Self-Anxiety, and SEMA.

\subsection{Cognitive and affective correlates of math}

In order to assess the unique contribution of each variable to math attainment, we ran a blockwise regression analysis with the math composite score as criterion variable (see Table 2). To assess whether anxiety significantly contributed to unique Math variance beyond the cognitive correlates, we entered the cognitive variables in the first block of the regression model, and the three anxiety variables in the second block. Because mild-to-moderate interrelations were expected and observed among the cognitive measures, a stepwise selection procedure was adopted within each block of the regression model in order to prevent multicollinearity effects, with $p \leq .05$ and $p \geq .10$ values as inclusion and exclusion criteria, respectively.

As detailed in Table 2, among the cognitive predictors inserted in Block I, Intelligence, Backward digit recall, Approximate addition, and 
Table 1

Descriptive statistics and inter-correlations between all variables.

\begin{tabular}{|c|c|c|c|c|c|c|c|c|c|c|c|c|c|c|c|c|c|}
\hline & & \multicolumn{6}{|c|}{ Descriptive statistics } & \multicolumn{10}{|c|}{ Zero-order correlations } \\
\hline & & Mean & $S D$ & Min & Max & Skewness & Kurtosis & 2. & 3. & 4. & 5. & 6. & 7. & 8. & 9. & 10. & 11. \\
\hline 1. & Intelligence & 10.82 & 2.63 & 3.00 & 18.50 & 0.01 & 0.30 & $.19^{*}$ & $.26^{\text {tik }}$ & .13 & $.23^{* * *}$ & .06 & .04 & .09 & -.05 & -.12 & $.33^{* * * *}$ \\
\hline 2. & Verbal WM (Backward word span) & 1.89 & 1.32 & .00 & 5.00 & 0.20 & -0.94 & - & $.39^{* \neq k}$ & .13 & $.16^{*}$ & .02 & .10 & -.06 & $-.16^{*}$ & -.01 & $.21^{k * *}$ \\
\hline 3. & Verbal WM (Backward digit span) & 3.85 & 1.25 & .00 & 7.00 & 0.02 & -0.23 & - & - & $.20^{* k+k}$ & $.16^{*}$ & .03 & .02 & -.02 & $-.26^{* *}$ & .07 & $.29^{* * k}$ \\
\hline 4. & Visuo-Spatial WM (Path dual task) & 6.27 & 1.71 & .00 & 8.00 & -1.29 & 1.73 & - & - & - & .10 & $.19^{*}$ & .03 & .07 & -.01 & .01 & $.21^{* * *}$ \\
\hline 5. & Estimation & 58.19 & 12.96 & 6.00 & 82.00 & -1.27 & 2.59 & - & - & - & - & $.27^{* * * e}$ & .06 & .12 & -.10 & -.05 & $.27^{k *}$ \\
\hline 6. & Approximate Comparison & 26.94 & 3.64 & 16.00 & 36.00 & -0.30 & 0.34 & - & - & - & - & - & $.26^{* * *}$ & .03 & -.11 & -.03 & .12 \\
\hline 7. & Approximate Addition & 17.60 & 2.67 & 7.00 & 23.00 & -1.07 & 1.73 & - & - & - & - & - & - & -.07 & $-.23^{* * *}$ & -.13 & $.25^{* * *}$ \\
\hline 8. & Anxiety-Self & 7.03 & 3.79 & .00 & 20.00 & 0.53 & -0.05 & - & - & - & - & - & - & - & .15 & $.37^{* * 2 * k}$ & $<.01$ \\
\hline 9. & Anxiety-Observed & 1.47 & 1.52 & .00 & 7.00 & 1.03 & 0.61 & - & - & - & - & - & - & - & - & .09 & $-.29^{k+k n}$ \\
\hline 10. & Math Anxiety & 14.44 & 11.00 & .00 & 57.00 & 0.89 & 0.86 & - & - & - & - & - & - & - & - & - & -.10 \\
\hline 11. & Composite Math Score & 7.71 & 2.75 & .00 & 12.00 & -0.77 & 0.28 & - & - & - & - & - & - & - & - & - & - \\
\hline
\end{tabular}

Note. Min $=$ minimum; $\operatorname{Max}=$ maximum; $S D=$ standard deviation.

${ }^{\dagger} p<.10$.

$* p \leq .05$.

$* * p \leq .01$.

$* * * p \leq .001$.

Table 2

Blockwise regression model predicting math performance from cognitive and affective factors.

\begin{tabular}{|c|c|c|c|c|c|c|}
\hline & $B$ & $S E(B)$ & $\beta$ & $p$ & $R^{2}$ & $\Delta R^{2}$ \\
\hline \multicolumn{7}{|l|}{ Block I - Cognitive Factors } \\
\hline Step 1 & & & & & $.11^{* * * * *}$ & - \\
\hline Intelligence & .11 & .03 & .33 & $<.001$ & & \\
\hline Step 2 & & & & & $.17^{* \ldots * x}$ & $.06^{* * x}$ \\
\hline Intelligence & .11 & .03 & .32 & $<.001$ & & \\
\hline Approximate addition & .08 & .03 & .24 & .001 & & \\
\hline Step 3 & & & & & $.21^{* * * *}$ & $.04^{* *}$ \\
\hline Intelligence & .09 & .03 & .24 & .002 & & \\
\hline Approximate addition & .08 & .02 & .23 & .002 & & \\
\hline Backward digit span & .15 & .05 & .22 & .005 & & \\
\hline Step 4 & & & & & $.24^{* * * *}$ & $.03^{*}$ \\
\hline Intelligence & .08 & .03 & .25 & .002 & & \\
\hline Approximate addition & .08 & .02 & .24 & .002 & & \\
\hline Backward digit span & .14 & .05 & .20 & .010 & & \\
\hline Estimation & .01 & .01 & .16 & .028 & & \\
\hline \multicolumn{7}{|c|}{ Block II - Affective Factors } \\
\hline Step 5 & & & & & $.27^{\text {****k}}$ & $.03^{*}$ \\
\hline Intelligence & .09 & .03 & .25 & .001 & & \\
\hline Approximate addition & .06 & .02 & .18 & .011 & & \\
\hline Backward digit span & .10 & .05 & .14 & .063 & & \\
\hline Estimation & .01 & .01 & .16 & .035 & & \\
\hline Anxiety-Observed & -.11 & .05 & -.18 & .010 & & \\
\hline
\end{tabular}

Note. $S E$ = standard error. Within each block, a stepwise procedure was adopted for variable selection. Inclusion criterion: $p \leq .05$; exclusion criterion: $\mathrm{p}>.10$.

$$
\begin{aligned}
& * p \leq .05 . \\
& * * p \leq .01 . \\
& * * * p \leq .001 .
\end{aligned}
$$

Estimation were entered in the model, whereas Backward word recall, Path dual task, and Approximate magnitude comparison were not. With the cognitive abilities only concurring to the determination of math score, the model accounted for $24 \%$ of the total variance. When adding the anxiety measures included in Block II, also Anxiety-Observed emerged as significant predictor and was retained in the model. The predictivity of the model further increased by $3 \%$, and the full model accounted for $27 \%$ of variance in Math. None of the previously retained cognitive abilities was removed from the model in subsequent steps of the analyses. However, as apparent from Table 2, the contribution of Backward digit recall, Approximate addition, and Estimation slightly reduced when Anxiety-Observed was entered in the model, thus suggesting that part of the variability in the math score was shared among cognitive and affective factors.

\subsection{Mediation analyses}

In order to test the hypothesis that deficiencies in both domaingeneral and domain-specific cognitive abilities may mediate the relation between anxiety and math performance, we ran a multiple mediation model using the analytical approach and the PROCESS macro for SPSS by Hayes [49]. This approach allows to simultaneously estimate total, direct, and indirect effects of the main predictor on the dependent variable through each proposed mediator. The point estimate and the confidence intervals (CI) for the indirect effects were estimated through a bias-corrected bootstrapping procedure with 10,000 resamples. Based on the blockwise regression results, only Backward digit recall, Approximate addition, and Estimation were retained as candidate mediators. Anxiety-Observed was modeled as the main predictor, and general Intelligence was retained as a covariate. Results of the mediation analysis are reported in Fig. 1.

The total effect of Anxiety-Observed $\left(B_{\text {TотаL }}=-.17, S E=.04, p=\right.$ .002 , 95\%CI: $-.26 ;-.08$ ), on Math was significant, but was markedly reduced when the three candidate mediators were inserted in the model $\left(B_{\text {DIRECT }}=-.11, S E=.04, p=.014,95 \% \mathrm{CI}:-.20 ;-.02\right)$. The paths linking Anxiety-Observed to Backward digit recall and Approximate addition-but not Estimation-were significant. Both the mediated paths linking Anxiety-Observed to Math through either Backward digit recall $\left(B_{\mathrm{INDIRECT}}=-.023, S E=.015,95 \% \mathrm{CI}:-.062 ;-.001\right)$ or Approximate addition $\left(B_{\text {INDIRECT }}=-.026, S E=.013,95 \% \mathrm{CI}\right.$ : $\left.-.061 ;-.006\right)$ were

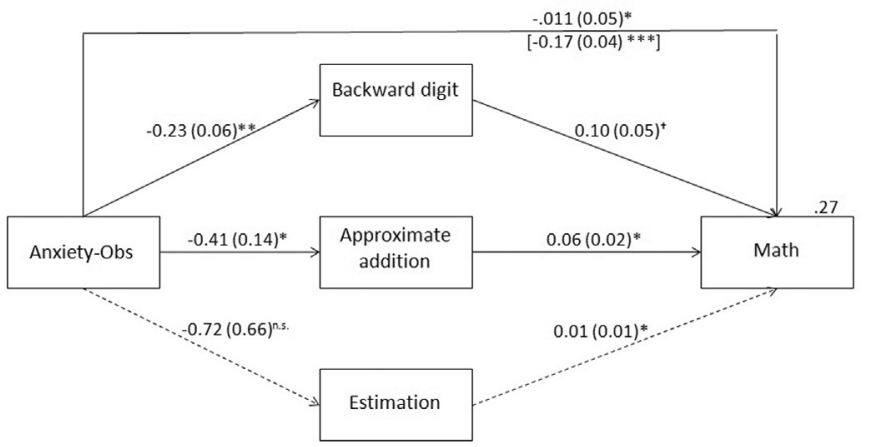

Fig. 1. Multiple Mediation Model. Unstandardized coefficient of the mediation model between general anxiety (Anxiety-Obs) and math achievement (Math) through backward digit memory, approximate addition, and estimation (standard errors presented in parentheses). In squared parentheses, the total effect (with standard error) of AnxietyObserved on Math. Dashed lines indicate non-significant paths. ${ }^{\dagger} p<.10,{ }^{*} p \leq .05$, ${ }^{* *} p \leq$ $.01, * * * p \leq .001$. 
significant, whereas the path through Estimation was not $\left(B_{\text {INDIRECT }}=\right.$ $-.008, S E=.009,95 \%$ CI: $-.034 ; .004$ ).

A plausible explanation for the present results is that anxiety may interfere with Approximate addition (and not Estimation) because the former task requires higher executive control than the latter, and therefore recruits WM resources. To evaluate this hypothesis, we ran a sequential mediation analysis; here, a two-step indirect path linking Anxiety-Observed to Math through Backward digit recall and, in sequence, Approximate addition, was added to the mediation model. However, results confirmed that both the simple indirect paths from Anxiety-Observed to Math through Backward digit span $\left(B_{\text {INDIRECT }}=\right.$ $-.023, S E=.015,95 \% \mathrm{CI}:-.061 ;-.001)$ and Approximate addition $\left(B_{\text {INIRECT }}=-.027, S E=.015,95 \% \mathrm{CI}:-.065 ;-.007\right)$ remained significant, whereas the sequential indirect path (i.e., Anxiety-Observed Backward digit span - Approximate addition - Math) was not ( $B_{\text {INDIRECT }}$ $=-.002, S E=.003,95 \% \mathrm{CI}:-.003 ; .011)$.

\section{Discussion}

The relation between anxiety and mathematics proficiency in very young students is still an underinvestigated issue, and the interplay between anxiety and the diverse cognitive abilities at the basis of math is even less explored. Whereas some studies focused on the link between anxiety and WM (e.g., $[97,96,111]$ ), or between anxiety and the domain-specific correlates of math abilities (e.g., [43]), to the best of our knowledge no single study to date simultaneously took into account both domain-general and domain-specific cognitive components, in order to observe whether and how they can be differentially associated with both general and math-specific anxiety. Our study was designed to fill these gaps, by trying to achieve a more exhaustive picture of cognitive and affective contributors of math achievement in young schoolchildren.

\subsection{Independent effects of cognitive and affective components on math performance}

Consistent with previous findings with older students (e.g., [82]), the results from the present study highlighted that already in young schoolchildren a significant negative relation could be traced between general anxiety and math performance. This outcome is important because it evidences how negative affective states may interfere with cognitive proficiency since the earliest primary school grades (e.g., $[18,70])$. Notably, the contribution of general anxiety remained significant even when controlling for both domain-general (i.e., WM and intelligence) and domain-specific (i.e., ANS) cognitive correlates of math. This finding also highlights that, all the other things being equal, general anxiety alone is sufficient to depress early math performance. Moreover, the size of the anxiety effect was almost comparable- - to the contribution of the single cognitive factors we examined.

It has however to be pointed out that the observed relation between anxiety and the other constructs of interest was limited to the measure of general anxiety, and more precisely to the teachers' proxy report of children's anxiety. This pattern of results is consistent with previous studies, in which the agreement between children's self-reports and the proxy reports of general anxiety from either parents' (e.g., [78,114]) or teachers' (e.g., $[7,78]$ ) was commonly quite low (see the recent metaanalysis by [22]). Teachers' capacity to detect the anxiety symptoms, when present, was repeatedly demonstrated (e.g., $[58,68,79,101]$ ), and the discrepancy with children's self-ratings was hypothesized to depend on the children's difficulty to appraise a multifaceted and complex construct such as general anxiety is.

Contrary to general anxiety, math-specific anxiety appeared to be unrelated with math performance. In this case, past evidence suggests that the lack of relation with performance is not likely to depend on the children's difficulty in answering the scale: Math anxiety is a specific construct that can be contextualized and reliably self-reported even by young children (see $[97,96,115,117]$; but see [36]). The absence of a significant relation between math anxiety and math performance is consistent with previous studies conducted by using the same measures with 7-year-old children in the same cultural context [18], but inconsistent with those reported by $\mathrm{Wu}$ et al. (2012) in a different school system. We may argue that the different pattern of results may depend, at least in part, on the fact that formal mathematical instruction is not prompted in Italy before entering primary school (at the age of 6); on the contrary, children in other school systems usually receive formal math instruction (and in some cases also formal evaluation) at a lower age (e.g., from 4 to 5 years of age in the UK). It is therefore plausible that math anxiety, although clearly detectable since the earliest school grades, could become significantly related to math performance only after a reasonably long experience with math instruction. Unfortunately, cross-cultural studies investigating children's math anxiety in different countries are not available to date, and more thorough research is needed to better clarify the onset of the detrimental association between anxiety and math performance in young schoolchildren. Future research adopting alternative approaches to the assessment of math-specific and general anxiety, such as physiological measures (see [70]), could help unraveling the issue.

As regards the cognitive factors contributing to math proficiency, our findings confirmed the involvement of both domain-general and domain-specific cognitive processes. However, in line with prior literature, the diverse components of both WM and ANS contributed to a different extent to formal math outcomes. In detail, even though the visuo-spatial component of WM was associated with math outcomes with an effect size comparable to that of the verbal memory components, it did not emerge as a significant predictor of math performance when other cognitive factors were controlled for. In line with previous evidence (e.g., $[23,52,76]$ ), it might be speculated that visuo-spatial WM could exert a chief role in the earliest stages of numeracy development. Conversely, when children begin to master robust verbal competence and spontaneous rehearsal strategies at around the age of 7, the visuo-spatial memory components may become relatively less important. However, the cross-sectional design of the present research does not allow a direct test of this conjecture. Therefore, future research should more deeply address the issue, also considering the fact that evidences exist in support to the involvement of visuo-spatial WM also in older children, both typically achieving (e.g. $[52,57,98]$ ) and with math disabilities (e.g., [88]).

With respect to the verbal WM component, a significant involvement emerged for the measure based on digit (but not word) recall, even though the two indices were moderately correlated. This pattern of results is in line with previous studies, in which a stronger association between WM and math emerged when numerical material was used to assess memory efficiency (e.g., [62,85,104]). However, other studies found similar associations between math and either numerical or non-numerical tasks testing verbal WM (e.g., [59,107]). Actually, there is lack of consensus with respect to the relation between diverse WM components and math skills. It is plausible for the different aspects of WM to have a differential impact on math in dependence of the specific math skill that is taken into account (for recent meta-analyses, see $[32,92])$. It appears therefore advisable for future studies to more deeply investigate the relation between different components of WM and math, with a further particular attention to the children's age.

As regards ANS, results revealed that only the tasks assessing approximate addition and estimation abilities were related to math proficiency, whereas no association emerged between the widely used dot comparison task and formal math. Further, the lack of strong intercorrelations between the diverse ANS measures may actually reflect the multifaceted nature of the innate ability to manipulate numerosities in an approximate manner.

In a developmental perspective, our results may indicate that some ANS measures (such as the dot comparison task) may lose importance when taking into account formal math learned in primary school, 
whereas others (such as approximate addition and estimation) may still be relevant. For instance, even though dot comparison is probably the most widely used task to assess ANS, consistent findings on its predictive role in math were actually found in very young children (e.g., $[65,74,84,118])$, whereas more conflicting evidence emerged from studies with older children (e.g., [53,102]). It could therefore happen that, through development and education, some abilities more than others may result to be relevant. This position is corroborated, for example, by Pinheiro-Chagas et al. [95], who found that the impact of approximate comparison on math performance in primary school children was mediated by the acuity in approximate addition and estimation. Unfortunately, very few studies to date combined diverse measures to assess ANS and its relation with formal math, and almost no work attempted an exploration of developmental changes in the associations between approximate and exact numerical abilities. Future research is therefore needed to address this gap.

\subsection{Relation between cognitive and affective components in determining math performance}

Beyond proving further evidence for the direct negative relation between general anxiety and math performance, the present study also attempted to understand whether anxiety may also indirectly disrupt math proficiency by negatively affecting the domain-general and the domain-specific cognitive correlates of math. In fact, whereas the negative influence of anxiety on the execution of tasks involving WM is well established (at least in older children and adults, see [29]), its impact on more automatic and basic quantitative skills, such as those related to non-symbolic ANS, is still underinvestigated. Our findings proved that-among the correlates that significantly predicted math proficiency-WM for digits and the approximate addition skills were both significant mediators of the relation between general anxiety and math, whereas estimation was not.

The finding concerning WM is in line with robust empirical evidence proving that high anxiety levels could deplete the WM resources at the basis of math (see [13], for a discussion). As a consequence, when children are faced with math tasks in presence of external factors-as anxiety-that reduce the WM resources, they are prompted to use less demanding but also less efficient strategies, hence leading to a poorer performance $[10,96]$.

The finding related to the mediating role of the ANS measure of approximate addition is indeed more innovative, given that the few studies targeting the relation between ANS and anxiety had different aims, and mainly focused on math anxiety rather than on general anxiety (e.g., $[43,47,112])$. Importantly, and different from our findings, the majority of prior studies did not observe any significant involvement of the ANS components, at least when expressed by non-symbolic skills.

Different explanations could account for our observed relation between general anxiety and approximate addition skills. One possibility calls for the involvement of the inhibition processes with which anxiety is known to interfere, as for instance contended by the inhibition mechanism theory [54]. As outlined by some scholars, inhibition may be involved in the execution of tasks tapping ANS, and by partialling out the contribution of inhibition, the predictive power of ANS on math was found to reduce or even disappear (e.g., [35,41,105]). In line with this reasoning, one may hypothesize that approximate addition mediates the relation between anxiety and math proficiency in the measure that anxiety depresses the inhibition skills that are necessary to perform the task. Unfortunately, no measure of inhibition was included in the present work, and future studies are needed to disentangle the possible role of inhibitory skills. However, in a more general vein, we took into account the possibility that approximate addition-but not estimation-exerted a mediating role between anxiety and math proficiency because the former task requires executive resources to be performed, whereas the latter does not. Results from a sequential mediation analysis, in which verbal WM (namely memory for digits) was inserted as a first-order mediator between anxiety and the approximate addition measure, did not support this contention, and confirmed that the two measures independently acted as mediators of the relation between general anxiety and math proficiency.

\section{Conclusion}

In sum, the outcomes of the present study extend previous literature in two directions. First, the negative relation between anxiety and math performance was confirmed also for very young students. Second, this relation was observed to operate through the disruption of some of both domain-general and domain-specific cognitive correlates of math abilities. Notably, also one task pertaining to ANS (i.e., Approximate addition) emerged as a crucial mediator of this relation and acted independently of the parallel depletion of the verbal WM resources.

In relation to anxiety, significant outcomes were confined to general anxiety, whereas math anxiety resulted roughly unrelated to both math performance and cognitive abilities. Future investigations are consequently necessary to inspect when math anxiety could begin to assume a significant role not only as a math predictor, but also as a factor able to deflate the cognitive abilities underlying math skills.

Results of the present study are however noteworthy from the educational viewpoint. By highlighting the involvement of the affective components in even very young students, these findings suggest that a coordinated evaluation of both affective and cognitive factors could be fundamental as an indicator of academic proficiency (in agreement with [110]). Hence, to avoid negative repercussions of poor math proficiency in different aspects of children's life, greater care has to be taken to precociously detect and treat not only deficiencies in cognitive math abilities-as traditional approaches do-but also fragilities occurring at the affective sphere.

\section{Acknowledgment}

This work was supported by the Grant Beneficentia Stiftung, 2016 to Maria Chiara Passolunghi.

\section{References}

[1] American Psychiatric Association, Diagnostic and Statistical Manual of Mental Disorders, 5th ed., Author, Washington, DC, 2013 (Text Revision).

[2] G. Amoretti, L. Bazzini, A. Pesci and M. Reggiani, MAT-2. Test di matematica. Firenze: GIUNTI O.S, 2007.

[3] M.H. Ashcraft, E.P. Kirk, The relationships among working memory, math anxiety, and performance, J. Exp. Psychol.: Gen. 130 (2001) 224-237, http://dx.doi.org/ 10.1037//0096-3445.130.2.224.

[4] U. Andersson, B. Lyxell, Working memory deficit in children with mathematical difficulties: a general or specific deficit? J. Exp. Child Psychol. 96 (2007) 197-228, http://dx.doi.org/10.1016/j.jecp.2006.10.001.

[5] A.D. Baddeley, Working Memory, Oxford University Press, Oxford, UK, 1986.

[6] A.D. Baddeley, G.J. Hitch, Working memory, in: G.A. Bower (Ed.), Recent Advances in Learning and Motivation, 8 Academic Press, New York, NY, 1974, pp. 47-89.

[7] J.S. Baldwin, M.R. Dadds, Reliability and validity of parent and child versions of the Multidimensional Anxiety Scale for Children in community samples, J. Am. Acad. Child Adolesc. Psychiatry 46 (2007) 252-260, http://dx.doi.org/10.1097/ 01.chi.0000246065.93200.a1.

[8] W.J. Barbaresi, S.K. Katusic, R.C. Colligan, A.L. Weaver, S.J. Jacobsen, Math learning disorder: incidence in a population-based birth cohort, 1976-82, Rochester, MN, Ambul. Pediatr. 5 (2005) 281-289, http://dx.doi.org/10.1367/ A04-209R.1.

[9] A.J. Baroody, M.R. Gatzke, The estimation of set size by potentially gifted kindergarten-age children, J. Res. Math. Educ. 22 (1991) 59-68, http://dx.doi.org/ $10.2307 / 749554$.

[10] P. Barrouillet, P. Lépine, Working memory and children's use of retrieval to solve addition problems, J. Exp. Child Psychol. 91 (2005) 183-204, http://dx.doi.org/ 10.1016/j.jecp.2005.03.002.

[11] D. Bartelet, A. Vaessen, L. Blomert, D. Ansari, What basic number processing measures in kindergarten explain unique variability in first-grade arithmetic proficiency? J. Exp. Child Psychol. 117 (2014) 12-28, http://dx.doi.org/10.1016/ j.jecp.2013.08.010.

[12] H. Barth, K. La Mont, J.S. Lipton, E.S. Spelke, Abstract number and arithmetic in 
preschool children, Proc. Natl. Acad. Sci. 102 (2005) 14116-14121, http://dx.doi. org/10.1073/pnas.0505512102.

[13] S.L. Beilock, Math performance in stressful situations, Curr. Dir. Psychol. Sci. 17 (2008) 339-343, http://dx.doi.org/10.1111/j.1467-8721.2008.00602.x.

[15] R. Bull, K.A. Espy, S.A. Wiebe, Short-term memory, working memory, and executive functioning in preschoolers: longitudinal predictors of mathematical achievement at age 7 years, Dev. Neuropsychol. 33 (2008) 205-228, http://dx. doi.org/10.1080/87565640 801982312.

[16] M. Cadinu, A. Maass, A. Rosabianca, J. Kiesner, Why do women underperform under stereotype threat? Evidence for the role of negative thinking, Psychol. Sci. 16 (2005) 572-578, http://dx.doi.org/10.1111/j.0956-7976.2005.01577.x.

[17] E. Carey, A. Devine, F. Hill, D. Szúcs, Differentiating anxiety forms and their role in academic performance from primary to secondary school, PLoS ONE 12 (2017) 1-20, http://dx.doi.org/10.1371/journal.pone.0174418.

[18] E. Cargnelutti, C. Tomasetto, M.C. Passolunghi, How is anxiety related to math performance in young students? A longitudinal study of Grade 2 to Grade 3 children, Cogn. Emot. 31 (2017) 755-764, http://dx.doi.org/10.1080/02699931. 2016.1147421.

[20] P.T. Cirino, The interrelationships of mathematical precursors in kindergarten, J. Exp. Child Psychol. 108 (2011) 713-733, http://dx.doi.org/10.1016/j.jecp.2010. 11.004.

[21] C. Cornoldi, T. Vecchi, Visuo-Spatial Working Memory and Individual Differences, Psychology Press, Hove, UK, 2003.

[22] A. De Los Reyes, T.M. Augenstein, M. Wang, S.A. Thomas, D.A.G. Drabick, D.E. Burgers, J. Rabinowitz, The validity of the multi-informant approach to assessing child and adolescent mental health, Psychol. Bull. 141 (2015) 858-900, http://dx.doi.org/10.1037/a0038498.

[23] B. De Smedt, R. Janssen, K. Bouwens, L. Verschaffel, B. Boets, P. Ghesquière, Working memory and individual differences in mathematics achievement: a longitudinal study from first grade to second grade, J. Exp. Child Psychol. 103 (2009) 186-201, http://dx.doi.org/10.1016/j.jecp.2009.01.004.

[24] B. De Smedt, M.-P. Noël, C. Gilmore, D. Ansari, How do symbolic and non-symbolic numerical magnitude processing skills relate to individual differences in children's mathematical skills? A review of evidence from brain and behavior, Trends Neurosci. Educ. 2 (2013) 48-55, http://dx.doi.org/10.1016/j.tine.2013. 06.001.

[25] S. Dehaene, V. Izard, and M. Piazza, Control over non-numerical parameters in numerosity experiments. Unpublished manuscript (available on 〈www.unicog. org $>$ ).

[27] C. Eden, A. Heine, A.M. Jacobs, Mathematics anxiety and its development in the course of formal schooling-a review, Psychology 4 (2013) 27-35, http://dx.doi. org/10.4236/psych.2013.46A2005.

[28] M.W. Eysenck, Anxiety: The Cognitive Perspective, Erlbaum, Hove, UK, 1992

[29] M.W. Eysenck, N. Derakshan, R. Santos, M.G. Calvo, Anxiety and cognitive performance: attentional control theory, Emotion 7 (2007) 336-353, http://dx.doi. org/10.1037/1528-3542.7.2.336.

[30] M.W. Eysenck, S. Payne, N. Derakshan, Trait anxiety, visuospatial processing, and working memory, Cogn. Emot. 19 (2005) 1214-1228, http://dx.doi.org/10.1080/ 02699930500260245.

[31] L.K. Fazio, D.H. Bailey, C.A. Thompson, R.S. Siegler, Relations of different types of numerical magnitude representations to each other and to mathematics achievement, J. Exp. Child Psychol. 123 (2014) 53-72, http://dx.doi.org/10.1016/j.jecp. 2014.01.013.

[32] I. Friso-van den Bos, S.H. van der Ven, E.H. Kroesbergen, J.E. van Luit, Working memory and mathematics in primary school children: a meta-analysis, Educ. Res. Rev. 10 (2013) 29-44, http://dx.doi.org/10.1016/j.edurev.2013.05.003.

[33] L.S. Fuchs, D.C. Geary, D.L. Compton, D. Fuchs, C.L. Hamlett, P.M. Seethaler, J.D. Bryant, C. Schatschneider, Do different types of school mathematics development depend on different constellations of numerical versus general cognitive abilities? Dev. Psychol. 46 (2010) 1731-1746, http://dx.doi.org/10.1037/ a0020662.

[34] L.S. Fuchs, D.C. Geary, D. Fuchs, D.L. Compton, C.L. Hamlett, Pathways to thirdgrade calculation versus word-reading competence: are they more alike or different? Child Dev. 87 (2016) 558-567, http://dx.doi.org/10.1111/cdev.12474.

[35] M. Fuhs, N. McNeil, ANS acuity and mathematics ability in preschoolers from lowincome homes: contributions of inhibitory control, Dev. Sci. 16 (2013) 136-148, http://dx.doi.org/10.1111/desc.12013.

[36] C.M. Ganley, A.L. McGraw, The development and validation of a revised version of the math anxiety scale for young children, Front. Psychol. 7 (2016) 1181, http:// dx.doi.org/10.3389/fpsyg.2016.01181.

[37] S.E. Gathercole, T.P. Alloway, Practitioner review: short-term and working memory impairments in neurodevelopmental disorders: diagnosis and remedial support, J. Child Psychol. Psychiatry 47 (2006) 4-15, http://dx.doi.org/10.1111/ j.1469-7610.2005.01446.x.

[38] D.C. Geary, Cognitive predictors of achievement growth in mathematics: a 5-year longitudinal study, Dev. Psychol. 47 (2011) 1539-1552, http://dx.doi.org/10 $1037 / \mathrm{a} 0025510$.

[39] D.C. Geary, M.K. Hoard, J. Byrd-Craven, M. Desoto, Strategy choices in simple and complex addition: contributions of working memory and counting knowledge for children with mathematical disability, J. Exp. Child Psychol. 88 (2004) 121-151, http://dx.doi.org/10.1016/j.jecp.2004.03.002.

[40] C. Gilmore, Approximate arithmetic in childhood, in: A. Dowker, R. Cohen Kadosh (Eds.), The Oxford Handbook of Mathematical Cognition, OUP, Oxford, UK, 2013, pp. 310-330, , http://dx.doi.org/10.1093/oxfordhb/9780199642342.013.006.

[41] C.K. Gilmore, N. Attridge, S. Clayton, L. Cragg, S. Johnson, N. Marlow, V. Simms, M. Inglis, Individual differences in inhibitory control, not non-verbal number acuity, correlate with mathematics achievement, PloS ONE 8 (2013) e67374, http://dx.doi.org/10.1371/journal.pone.0067374.

[42] P. Giovanardi Rossi and T. Malaguti, Valutazione delle abilità matematiche. Analisi dei livelli di apprendimento e dei disturbi specifici. Guida per l'insegnante (Assessment of math abilities. Guide for teachers). Trento: Centro Studi Erickson, 1994.

[43] F.R. Gómez-Velázquez, G. Berumena, A.A. González-Garrido, Comparisons of numerical magnitudes in children with different levels of mathematical achievement. An ERP study, Brain Res. 1627 (2015) 189-200, http://dx.doi.org/10.1016/ j.brainres.2015.09.009.

[44] W.S. Grolnick, R.M. Ryan, Self-perceptions, motivation, and adjustment in chil dren with learning disabilities: a multiple group comparison study, J. Learn. Disabil. 23 (1990) 177-184.

[45] J. Halberda, R. Ly, J.B. Wilmer, D.Q. Naiman, L. Germine, Number sense across the lifespan as revealed by a massive Internet-based sample, Proc. Natl. Acad. Sci. 109 (2012) 11116-11120, http://dx.doi.org/10.1073/pnas.1200196109.

[46] J. Halberda, M.M.M. Mazzocco, L. Feigenson, Individual differences in non-verbal number acuity correlate with math achievement, Nature 455 (2008) 665-668, http://dx.doi.org/10.1038/nature07246.

[47] S.A. Hart, J.A.R. Logan, L. Thompson, Y. Kovas, G. McLoughlin, S.A. Petrill, A latent profile analysis of math achievement, numerosity, and math anxiety in twins, J. Educ. Psychol. 108 (2016) 181-193, http://dx.doi.org/10.1037/ edu0000045.

[49] A.F. Hayes, The PROCESS macro for SPSS and SAS, 2015.

[50] R. Hembree, The nature, effects, and relief of mathematics anxiety, J. Res. Math Educ. 21 (1990) 33-46, http://dx.doi.org/10.2307/749455.

[51] G.J. Hitch, E. McAuley, Working memory in children with specific arithmetical learning difficulties, Br. J. Psychol. 82 (1991) 375-386.

[52] J. Holmes, J.W. Adams, Working memory and children's mathematical skills: implications for mathematical development and mathematics curricula, Educ. Psychol. 26 (2006) 339-366, http://dx.doi.org/10.1080/01443410500341056.

[53] I.D. Holloway, D. Ansari, Mapping numerical magnitudes onto symbols: the numerical distance effect and individual differences in children's mathematics achievement, J. Exp. Child Psychol. 103 (2009) 17-29, http://dx.doi.org/10. 1016/j.jecp.2008.04.001.

[54] D.R. Hopko, M.H. Ashcraft, J. Gute, K.J. Ruggiero, C. Lewis, Mathematics anxiety and working memory: support for the existence of a deficient inhibition mechanism, J. Anxiety Disord. 12 (1998) 343-355, http://dx.doi.org/10.1016/ S0887-6185(98)00019-X.

[55] D.C. Hyde, S. Khanum, E.S. Spelke, Brief non-symbolic, approximate number practice enhances subsequent exact symbolic arithmetic in children, Cognition 131 (2014) 92-107, http://dx.doi.org/10.1016/j.cognition.2013.12.007.

[56] T. Iuculano, J. Tang, C.W.B. Hall, B. Butterworth, Core information processing deficits in developmental dyscalculia and low numeracy, Dev. Sci. 11 (2008) 669-680, http://dx.doi.org/10.1111/j.1467- 7687.2008.00716. x.

[57] H.L. Jarvis, S.E. Gathercole, Verbal and nonverbal working memory and achievements on national curriculum tests at 7 and 14 years of age, Educ. Child Psychol. 20 (2003) 123-140.

[58] P.C. Kendall, A.C. Puliafico, A.J. Barmish, M.S. Choudhury, A. Henin, K.S. Treadwell, Assessing anxiety with the child behavior checklist and the teacher report form, J. Anxiety Disord. 21 (2007) 1004-1015, http://dx.doi.org/10.1016/ j.janxdis.2006.10.012.

[59] K.L. Koontz, D.B. Berch, Identifying simple numerical stimuli: processing inefficiencies exhibited by arithmetic learning disabled children, Math. Cogn. 2 (1996) 1-23, http://dx.doi.org/10.1080/135467996387525.

[60] K. Krajewski, W. Schneider, Exploring the impact of phonological awareness, visual-spatial working memory, and preschool quantity-number competencies on mathematics achievement in elementary school: findings from a 3-year longitudinal study, J. Exp. Child Psychol. 103 (2009) 516-531, http://dx.doi.org/10. 1016/j.jecp.2009.03.009.

[61] H. Krinzinger, L. Kaufmann, K. Willmes, Math anxiety and math ability in early primary school years, J. Psychoeduc. Assess. 27 (2009) 206-225, http://dx.doi. org/10.1177/0734282908330583.

[62] K. Landerl, A. Bevan, B. Butterworth, Developmental dyscalculia and basic numerical capacities: a study of 8-9-year-old students, Cognition 93 (2004) 99-125, http://dx.doi.org/10.1016/j.cognition.2003.11.004.

[63] S. Lanfranchi, C. Cornoldi, R. Vianello, Verbal and visuo-spatial working memory deficits in children with Down syndrome, Am. J. Ment. Retard. 109 (2004) 456-466, http://dx.doi.org/10.1352/0895-8017(2004)109.

[64] A.E. Layne, G.A. Bernstein, J.S. March, Teacher awareness of anxiety symptoms in children, Child Psychiatry Hum. Dev. 36 (2006) 383-392, http://dx.doi.org/10. 1007/s10578-006-0009-6.

[65] M.E. Libertus, L. Feigenson, J. Halberda, Preschool acuity of the approximate number system correlates with school math ability, Dev. Sci. 14 (2011) 1292-1300, http://dx.doi.org/10.1111/j.1467-7687.2011.01080.x.

[66] S. Linsen, L. Verschaffel, B. Reynvoet, B. De Smedt, The association between numerical magnitude processing and mental versus algorithmic multi-digit subtraction in children, Learn. Instr. 35 (2015) 42-50, http://dx.doi.org/10.1016/j. learninstruc.2014.09.003.

[67] S.F. Lourenco, J.W. Bonny, E.P. Fernandez, S. Rao, Nonsymbolic number and cumulative area representations contribute shared and unique variance to symbolic math competence, Proc. Natl. Acad. Sci. USA 109 (2012) 18737-18742, http://dx.doi.org/10.1073/pnas.1207212109.

[68] H.J. Lyneham, A.K. Street, M.J. Abbott, R.M. Rapee, Psychometric properties of the School Anxiety Scale-Teacher Report (SAS-TR), J. Anxiety Disord. 22 (2008) 292-300, http://dx.doi.org/10.1016/j.janxdis.2007.02.001. 
[70] M.A. MacKinnon McQuarrie, L.S. Siegel, N.E. Perry, J. Weinberg, Reactivity to stress and the cognitive components of math disability in grade 1 children, J. Learn. Disabil. 47 (2014) 349-365, http://dx.doi.org/10.1177/ 0022219412463436.

[71] E.A. Maloney, D. Ansari, J.A. Fugelsang, The effect of mathematics anxiety on the processing of numerical magnitude, Q. J. Exp. Psychol. 64 (2011) 10-16, http:// dx.doi.org/10.1080/17470218.2010.533278.

[72] E.A. Maloney, E.F. Risko, D. Ansari, J. Fugelsang, Mathematics anxiety affects counting but not subitizing during visual enumeration, Cognition 114 (2010) 293-297, http://dx.doi.org/10.1016/j.cognition.2009.09.013.

[73] I.C. Mammarella, F. Hill, A. Devine, S. Caviola, D. Szúcs, Math anxiety and developmental dyscalculia: a study on working memory processes, J. Clin. Exp. Neuropsychol. 37 (2015) 878-887, http://dx.doi.org/10.1080/13803395.2015. 1066759.

[74] M.M.M. Mazzocco, L. Feigenson, J. Halberda, Preschoolers' precision of the approximate number system predicts later school mathematics performance, PloS ONE 6 (2011) e23749, http://dx.doi.org/10.1371/journal.pone.0023749.

[75] M. McCloskey, Quantitative literacy and developmental dyscalculias, in: D.B. Berch, M.M.M. Mazzocco (Eds.), Why is Math so Hard for Some Children? The Nature and Origins of Children's Mathematical Learning Difficulties and Disabilities, Brookes Publishers, Baltimore, MD, 2007.

[76] B. McKenzie, R. Bull, C. Gray, The effects of visual-spatial and phonological disruption on children's arithmetical skills, Educ. Child Psychol. 20 (2003) 93-108.

[77] M.L. Meyer, V.N. Salimpoor, S.S. Wu, D.C. Geary, V. Menon, Differential contribution of specific working memory components to mathematics achievement in 2nd and 3rd graders, Learn. Individ. Differ. 20 (2010) 101-109, http://dx.doi.org/ 10.1016/j.lindif.2009.08.004.

[78] L.D. Miller, I.J. Martinez, E. Shumka, H. Baker, Multiple informant agreement of child, parent, and teacher ratings of child anxiety within community samples, Can. J. Psychiatry 59 (2014) 34-39.

[79] P.L. Newcomer, E.M. Barenbaum, B.R. Bryant, Depression and Anxiety in Youth Scale, PRO-ED, Austin, TX, 1994.

[80] N. Nosworthy, S. Bugden, L. Archibald, B. Evans, D. Ansari, A two-minute paperand-pencil test of symbolic and nonsymbolic numerical magnitude processing explains variability in primary school children's arithmetic competence, PloS One 8 (2013) e67918, http://dx.doi.org/10.1371/journal.pone.0067918.

[81] M.I. Núñez-Peña, M. Suárez-Pellicioni, Less precise representation of numerical magnitude in high math-anxious individuals: an ERP study of the size and distance effects, Biol. Psychol. 103 (2014) 176-183, http://dx.doi.org/10.1016/j. biopsycho.2014.09.004.

[82] M. Owens, J. Stevenson, J.A. Hadwin, R. Norgate, Anxiety and depression in academic performance: an exploration of the mediating factors of worry and working memory, Sch. Psychol. Int. 33 (2012) 443-449, http://dx.doi.org/10. 1177/0143034311427433.

[83] M.C. Passolunghi, S. Caviola, R. De Agostini, C. Perin, I.C. Mammarella, Mathematics anxiety, working memory, and mathematics performance in secondary-school children, Front. Psychol. 7 (2016) 42, http://dx.doi.org/10.3389/ fpsyg.2016.00042.

[84] M.C. Passolunghi, E. Cargnelutti, M. Pastore, The contribution of general cognitive abilities and Approximate Number System (ANS) in early mathematics, Br. J. Educ. Psychol. 84 (2014) 631-649, http://dx.doi.org/10.1111/bjep.12054.

[85] M.C. Passolunghi, C. Cornoldi, Working memory failures in children with arithmetical difficulties, Child Neuropsychol. 1 (2008) 1-14, http://dx.doi.org/10. 1080/09297040701566662.

[86] M.C. Passolunghi, S. Lanfranchi, Domain-specific and domain-general precursors of mathematical achievement: a longitudinal study from kindergarten to first grade, Br. J. Educ. Psychol. 82 (2012) 42-63, http://dx.doi.org/10.1111/j.2044 8279.2011.02039.x.

[87] M.C. Passolunghi, S. Lanfranchi, G. Altoè, N. Sollazzo, Early numerical abilities and cognitive skills in kindergarten children, J. Exp. Child Psychol. 135 (2015) 25-42, http://dx.doi.org/10.1016/j.jecp.2015.02.001.

[88] M.C. Passolunghi, I.C. Mammarella, Selective spatial working memory impairment in a group of children with mathematics learning disabilities and poor problemsolving skills, J. Learn. Disabil. 45 (2012) 341-350, http://dx.doi.org/10.1177/ 0022219411400746.

[89] M.C. Passolunghi, L.S. Siegel, Short-term memory, working memory, and inhibitory control in children with specific arithmetic learning disabilities, J. Exp. Child Psychol. 80 (2001) 44-57, http://dx.doi.org/10.1006/jecp.2000.2626.

[90] M.C. Passolunghi, L.S. Siegel, Working memory and access to numerical information in children with disability in mathematics, J. Exp. Child Psychol. 88 (2004) 348-367, http://dx.doi.org/10.1016/j.jecp.2004.04.002.

[91] D.J. Paulsen, M.G. Woldorff, E.M. Brannon, Individual differences in non-verbal number discrimination correlate with Event-related Potentials and measures of probabilistic reasoning, Neuropsychologia 48 (2010) 3687-3695, http://dx.doi. org/10.1016/j.neuropsychologia.2010.08.014.

[92] P. Peng, J. Namkung, M. Barnes, C. Sun, A meta-analysis of mathematics and working memory: moderating effects of working memory domain, type of mathematics skill, and sample characteristics, J. Educ. Psychol. 108 (2016) 455-473, http://dx.doi.org/10.1037/edu0000079.

[93] M. Piazza, A. Facoetti, A.N. Trussardi, I. Berteletti, S. Conte, D. Lucangeli, S. Dehaene, M. Zorzi, Developmental trajectory of number acuity reveals a severe impairment in developmental dyscalculia, Cognition 116 (2010) 33-41, http://dx. doi.org/10.1016/j.cognition.2010.03.012.

[94] M. Piazza, V. Izard, P. Pinel, D. Le Bihan, S. Dehaene, Tuning curves for approximate numerosity in the human intraparietal sulcus, Neuron 44 (2004) 547-555.

[95] P. Pinheiro-Chagas, G. Wood, A. Knops, H. Krinzinger, J. Lonnemann, I. StarlingAlves, K. Willmes, V.G. Haase, In how many ways is the Approximate Number System associated with exact calculation? PLoS ONE (2014) e111155, http://dx. doi.org/10.1371/journal.pone.0111155.

[96] G. Ramirez, H. Chang, E.A. Maloney, S.C. Levine, S.L. Beilock, On the relationship between math anxiety and math achievement in early elementary school: the role of problem solving strategies, J. Exp. Child Psychol. 141 (2016) 83-100, http://dx. doi.org/10.1016/j.jecp.2015.07.014.

[97] G. Ramirez, E.A. Gunderson, S.C. Levine, S.L. Beilock, Math anxiety, working memory and math achievement in early elementary school, J. Cogn. Dev. 14 (2013) 187-202, http://dx.doi.org/10.1080/15248372.2012.664593.

[98] M. Reuhkala, Mathematical Skills in Ninth-graders: relationship with visuo-spatial abilities and working memory, Educ. Psychol. 21 (2001) 387-399, http://dx.doi. org/10.1080/01443410120090786.

[100] O. Rubinsten, R. Tannock, Mathematics anxiety in children with developmental dyscalculia, Behav. Brain Funct. 6 (2010) 46, http://dx.doi.org/10.1186/17449081-6-46.

[101] H. Salbach-Andrae, N. Klinkowski, K. Lenz, U. Lehmkuhl, Agreement between youth-reported and parent-reported psychopathology in a referred sample, Eur. Child Adolesc. Psychiatry 18 (2009) 136-143, http://dx.doi.org/10.1007/ s00787-008-0710-z.

[102] D. Sasanguie, B. De Smedt, E. Defever, B. Reynvoet, Association between basic numerical abilities and mathematics achievement, Br. J. Dev. Psychol. 30 (2012) 344-357, http://dx.doi.org/10.1111/j.2044-835X.2011.02048.x.

[103] J.M. Sattler, Assessment of Children's Intelligence, Academic Press, San Diego, CA, 1992.

[104] L. Siegel, N. Pinkerton, L. Ford and A. Anderson, The cognitive components of a mathematical disability: The influence of definitional variables, in: Proceedings of the 36th Annual IARLD Conference, Padua, Italy, 2012.

[105] F. Soltész, D. Szúcs, L. Szúcs, Relationships between magnitude representation, counting and memory in 4- to 7-year-old children: a developmental study, Behav. Brain Funct. 6 (2010) 1-14, http://dx.doi.org/10.1186/1744-9081-6-13.

[106] R. Sorvo, T. Koponen, H. Viholainen, T. Aro, Räikkönen, P. Peura, A. Dowker, M. Aro, Math anxiety and its relationship with basic arithmetic skills among primary school children, Br. J. Educ. Psychol. (2017), http://dx.doi.org/10.1111/ bjep. 12151.

[107] C.M. Temple, S. Sherwood, Representation and retrieval of arithmetical facts: developmental difficulties, Q. J. Exp. Psychol. 55A (2002) 733-752, http://dx.doi. org/10.1080/02724980143000550.

[108] G. Thomas and A. Dowker, Mathematics anxiety and related factors in young children. Paper presented at British Psychological Society Developmental Section Conference, Bristol (UK), 2000.

[109] S.W.M. Toll, S. Van Viersen, E.H. Kroesbergen, J.E.H. Van Luit, The development of (non-)symbolic comparison skills throughout kindergarten and their relations with basic mathematical skills, Learn. Individ. Differ. 38 (2015) 10-17, http://dx. doi.org/10.1016/j.lindif.2014.12.006.

[110] K. Trezise, R.A. Reeve, Working memory, worry, and algebraic ability, J. Exp. Child Psychol. 121 (2014) 120-136, http://dx.doi.org/10.1016/j.jecp.2013.12. 001.

[111] R.K. Vukovic, M.J. Kieffer, S.P. Bailey, R.R. Harari, Mathematics anxiety in young children: concurrent and longitudinal associations with mathematical performance, Contemp. Educ. Psychol. 38 (2013) 1-10 (doi:10.1016=j.cedpysch.2012.09.001).

[112] Z. Wang, S.L. Lukowski, S.A. Hart, I.M. Lyons, L.A. Thompson, Y. Kovas, M.M.M. Mazzocco, R. Plomin, S.A. Petrill, Is math anxiety always bad for math learning? The role of math motivation, Psychol. Sci. 26 (2015) 1863-1876, http:// dx.doi.org/10.1177/0956797615602471.

[113] D. Wechsler, WISC-III. Wechsler Intelligent Scale for Children, Third edition, Giunti O. S, Firenze, 1991 (Italian Edition (2006)).

[114] M. Weeks, R.J. Coplan, A. Kingsbury, The correlates and consequences of early appearing social anxiety in young children, J. Anxiety Disord. 23 (2009) 965-972, http://dx.doi.org/10.1016/j.janxdis.2009.06.006.

[115] S.S. Wu, M. Barth, H. Amin, V. Malcame, V. Menon, Math anxiety in second and third graders and its relation to mathematics achievement, Front. Psychol. 3 (2012) 1-11, http://dx.doi.org/10.3389/fpsyg.2012.00162.

[116] S. Wu and V. Menon. Scale for Early Mathematics Anxiety (SEMA) in Young Children. Available at: 〈http://mathbrain.stanford.edu/?q= publications $\rangle$.

[117] S.S. Wu, E.G. Willcutt, E. Escovar, V. Menon, Mathematics achievement and anxiety and their relation to internalizing and externalizing behaviors, J. Learn. Disabil. 47 (2014) 503-514, http://dx.doi.org/10.1177/0022219412473154.

[118] I. Xenidou-Dervou, B. De Smedt, M. van der Schoot, E.C.D.M. van Lieshout, Individual differences in kindergarten math achievement: the integrative roles of approximation skills and working memory, Learn. Individ. Differ. 28 (2013) 119-129, http://dx.doi.org/10.1016/j.lindif.2013.09.012.

[119] C.B. Young, S.S. Wu, V. Menon, The neurodevelopmental basis of math anxiety, Psychol. Sci. 23 (2012) 492-501, http://dx.doi.org/10.1177/0956797611429134. 